Published in final edited form as:

Nucl Instrum Methods Phys Res A. 2011 August 21; 648(Suppl 1): S220-S223. doi:10.1016/j.nima. 2010.11.147.

\title{
Design and feasibility studies of a stationary digital breast tomosynthesis system
}

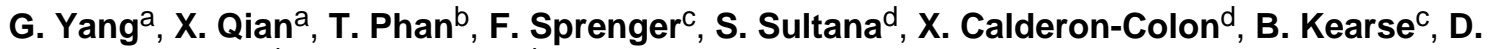 \\ Spronk $^{\mathrm{c}}$, J. Lu ${ }^{\mathrm{a}, \mathrm{d}}$, and $\mathbf{O}$. Zhou , $^{\mathrm{a}, \mathrm{d}, \mathrm{e}}$ \\ aDepartment of Physics and Astronomy, University of North Carolina at Chapel Hill, Chapel Hill, \\ NC 27599, USA \\ ${ }^{b}$ Department of Biomedical Engineering, University of North Carolina at Chapel Hill, Chapel Hill, \\ NC 27599, USA \\ 'XinRay Systems LLC, Research Triangle Park, NC 27709, USA \\ ${ }^{\mathrm{d} C}$ Curriculum in Applied Sciences and Engineering, University of North Carolina at Chapel Hill, \\ Chapel Hill, NC 27599, USA \\ eLineberger Comprehensive Cancer Center, University of North Carolina at Chapel Hill, Chapel \\ Hill, NC 27599, USA
}

\begin{abstract}
Studies have shown that digital breast tomosynthesis (DBT) can improve breast cancer diagnosis by reconstructing 3D images. However, DBT scanners based on rotation gantry prolong the imaging time and reduce spatial resolution due to motion comparing with the regular two-view mammography. To obtain three dimension reconstruction images and maintain the high image quality of conventional mammography, we proposed a prototype stationary digital breast tomosynthesis system (s-DBT). The proposed s-DBT system acquires projection images without mechanical movement. The core component of the s-DBT system is a specially designed spatially distributed multi-beam x-ray tube based on the carbon nanotube field emission x-ray technology. The multi-beam $\mathrm{x}$-ray source array enables collection of all projection images from different viewing angles without mechanical motion. Preliminary results show the s-DBT system can achieve a scan time comparable to the regular two-view mammography, and improve the spatial resolution comparing with rotating gantry DBT.
\end{abstract}

\section{Keywords}

digital breast tomosynthesis; carbon nanotube; field emission

\section{Introduction}

Digital breast tomosynthesis (DBT) [1] is a three dimensional imaging technique which reconstructs slice images using projections from a certain angular range. DBT can potentially improve the tumor conspicuity over conventional mammography by reducing

\footnotetext{
(C) 2010 Elsevier B.V. All rights reserved.

Publisher's Disclaimer: This is a PDF file of an unedited manuscript that has been accepted for publication. As a service to our customers we are providing this early version of the manuscript. The manuscript will undergo copyediting, typesetting, and review of the resulting proof before it is published in its final citable form. Please note that during the production process errors may be discovered which could affect the content, and all legal disclaimers that apply to the journal pertain.
} 
tissue overlapping [2]. Current DBT scanners are all based on single x-ray source full-field digital mammography (FFDM) systems. To acquire projection images from different angular positions, the x-ray gantry rotates during the scanning. The total scan time is much longer than exposure time of conventional mammography. The prolonged imaging time introduces patient motion blur, and the effective x-ray focal spot size is enlarged to $1 \mathrm{~mm}$ [3] or larger due to the x-ray source movement during exposure. Both effects degrade the image quality, which limits further improvement on breast cancer diagnosis.

In this study, we proposed and demonstrated a stationary DBT prototype scanner, which can overcome some of the limitations of the current DBT scanners. The design and preliminary test result are reported. Potential clinical applications are discussed.

\section{Material and methods}

\subsection{Stationary digital breast tomosynthesis}

Current DBT scanners require the physical movement of the x-ray tube, which is the limiting factor in the scanning speed. To eliminate the gantry motion, we proposed the concept of stationary digital breast tomosynthesis or s-DBT scanner [4-5]. As illustrated in Fig. 1, the s-DBT system is composed of a stationary x-ray source array, control electronics for the x-ray source, a flat panel detector, a detector controller, and a computer workstation. The $x$-ray source array replaces the rotation gantry. The x-ray source array consists of spatially distributed $\mathrm{x}$-ray beams which are individually addressable. The switching of each $\mathrm{x}$-ray beam is achieved through the control electronics and the computer workstation. The $\mathrm{x}$ ray radiation is synchronized with the detector readout with accuracy better than $1 \mathrm{~ms}$.

During a tomosynthesis imaging, projection x-ray images from different angles are acquired sequentially by switching the x-ray beams one by one. By eliminating the gantry movement, the s-DBT scanner can reduce the scan time and decrease the patient motion blur. The x-ray focal spot enlargement induced by rotating motion is also eliminated thanks to the stationary design. The image quality can be improved comparing to the DBT scanners with rotation gantry.

\subsection{Field emission x-ray source array based on carbon nanotubes}

A novel field emission $\mathrm{x}$-ray source technology based on carbon nanotube is applied in the proposed s-DBT system. The design of the cathode structure and electron beam optics to generate a single $\mathrm{x}$-ray beam is similar to the microfocus $\mathrm{x}$-ray source developed for a micro-CT system [6]. The schematic is shown in Fig. 2. The x-ray source is composed of a carbon nanotube cathode, a gate electrode, a focusing unit, and an anode. Carbon nanotube field emitters are deposited at a predefined small area on the substrate. The emission current is proportional to the cathode size and follows the Fowler-Nordheim equation [7]. Electrons are emitted under a bias gate voltage:

$$
I=a V^{2} \exp \left(-b \phi^{3 / 2} / V\right)
$$

where $a$ and $b$ are constants, and $\phi$ is the work function of the field emitter.

By using modified asymmetrical Einzel-type focusing lenses, the trajectory of the electrons can be well controlled. A demagnification from the cathode size to focal spot size up to 5 has been demonstrated [6].

Comparing to conventional thermionic cathodes, the novel $\mathrm{x}$-ray source has several intrinsic advantages, including high temporal resolution and the capabilities of spatial and temporal 
modulations. The feasibility of field emission x-ray source array based on carbon nanotubes has been studied [8]. The tomosynthesis imaging has been demonstrated with the prototype system with five $\mathrm{x}$-ray beams.

\section{Results}

\subsection{Bench-top prototype s-DBT scanner}

A bench-top s-DBT scanner has been developed [4]. Fig. 3 shows the system schematic with CAD drawing of the $\mathrm{x}$-ray source array. This prototype system consists of a linear $\mathrm{x}$-ray source array with 25 individual beams, a flat panel detector (model: PaxScan 2520, Varian Medical Systems, Salt Lake City, Utah USA), and control electronics. The source-todetector distance is $69.6 \mathrm{~cm}$, which is comparable to conventional mammography scanners. To fully utilize the $\mathrm{x}$-ray radiation, the $25 \mathrm{x}$-ray beams are tilted towards the system isocenter, which coincides with the center of the breast phantom. The angular coverage of the $25 \mathrm{x}$-ray beams is $48^{\circ}$ with $2^{\circ}$ separation between projections. A LabView program has been developed to control the scanner and acquire projection images. The s-DBT scanner has been fully characterized. System calibration result was reported earlier [9], including spectrum measurement, focal spot size, and geometry calibration.

Due to the performance variation, the required gate voltages are different to generate same currents from the 25 beams. To compensate the gate voltages, 25 variable resistors were integrated in the control circuit. To obtain constant X-ray output across all 25 beams, I V curves of the 25 cathodes were measured and resistor values were adjusted prior to imaging.

A Stereotactic needle biopsy tissue equivalent breast phantom (Model 013, Computerized Imaging Reference Systems, Inc., Norfolk, Virginia USA) was imaged on the prototype system. Anode voltage was $28 \mathrm{kVp}$, filter/target combination was $\mathrm{Mo} / \mathrm{Mo}$, and the total exposure level was $100 \mathrm{mAs}$. The images were reconstructed to 60 slices (slice thickness of 1 $\mathrm{mm})$ of size $1200 \times 700(0.1 \times 0.1 \mathrm{~mm})$ using Modified Ordered Subsets Convex (MOSC) algorithm. The slices shown in Fig. 4 are $3 \mathrm{~mm}$ apart and clearly show the various masses getting focused at different depths.

The spatial distribution of the x-ray beams is different from the s-DBT systems based on rotation gantry. Several other reconstruction algorithms, such as filtered back projection (FBP) and matrix inversion tomosynthesis (MITS), have also been modified to take into account the linear source geometry in the s-DBT system. Comparison between different algorithms has been reported [10].

\subsection{Prototype s-DBT scanner for clinical application}

To further investigate the clinical potential of the s-DBT, a second prototype scanner is under construction (Fig. 5). The flat panel detector is place horizontally and the x-ray source array is above the detector. The x-ray source array and detector can be rotated and fixed at desired angles for MLO view imaging. Additional collimators and shielding are also designed to meet FDA regulations [11]. The same control electronic system will be used on the new s-DBT scanner.

The other objective of the new scanner is to reduce the scan time. Because the field emission current is proportional to the cathode emission area, larger cathodes are fabricated for the new scanner to obtain higher x-ray tube current. Fig. 6 shows the emission current measurement result of 19 cathodes with $2 \mathrm{~mm} \times 8 \mathrm{~mm}$ emission area. Two cathodes have lower performance (broken lines in the diagram) and these cathodes will be screened out. The other 17 cathodes (solid lines in the diagram) can generate $30 \mathrm{~mA}$ current at electric field less than $7 \mathrm{~V} / \mu \mathrm{m}$. As the transmission rate (the ratio of anode current to cathode 
current) is usually $70 \%$ [9], the anode current can reach $20 \mathrm{~mA}$. The new s-DBT scanner can reduce the scan time to $6 \mathrm{~s}$ per scan based on $100 \mathrm{mAs}$ dose level, (most full field digital mammography and DBT scans require less than $100 \mathrm{mAs}$ radiation dose [12]).

\section{Conclusion and discussion}

A bench-top s-DBT scanner has been successfully built and characterized. The preliminary result shows the s-DBT design can potentially reduce the scan time and improve the image quality. With further optimization of electron beam focusing structure, it is possible to increase anode current to $60 \mathrm{~mA}$, this will reduce the scan time further to be around $2 \mathrm{~s}$, comparable with scan time of typical 2-view mammography. The second prototype s-DBT scanner has been designed for clinical studies. The system is capable for both CC and MLO scans. It only takes 10 seconds to change the projection mode. The system will be characterized and compared with other DBT systems.

The s-DBT system enables some advanced imaging techniques for breast imaging. The spatially distributed $\mathrm{x}$-ray sources are ideal for dual energy tomosynthesis imaging. With conventional DBT systems, dual energy imaging requires two scans. The time delay between the two scans may introduce noise and reduce image quality. With the s-DBT systems, the x-ray sources can be tuned at two energy levels. This can be achieved by either applying different anode voltages or filtering $\mathrm{x}$-ray beams with different materials. The proposed schematic is that $\mathrm{x}$-ray beams at odd numbers are tuned at low energy and $\mathrm{x}$-ray beams at even numbers are tuned at high energy. In such a design, a single scan can produce both 3D and energy dispersive images. The low and high energy tomosynthesis images will be first processed separately, then combined to resolve dual energy information.

The s-DBT system can also take advantage of the multiplexing technique to further reduce the scan time [13]. By simultaneously acquiring multiple projection images, the total scan time can be reduced up to a factor of N/2, where $\mathrm{N}$ is the number of $\mathrm{x}$-ray beams. Because of the reduction of the scan time, the s-DBT system makes quasi-monochromatic imaging feasible. This could potentially reduce the patient dose and improve image quality. These techniques will be studied with the new s-DBT system.

\section{Acknowledgments}

The study was partially supported by NCI Grant No. U54CA119343, NIH R01 Grant No. CA134598-01 and the University Cancer Research Fund at the University of North Carolina. One of the authors, Dr. Xin Qian, was supported by a fellowship from DoD Grant No. BC087505.

\section{References}

1. Dobbins JT 3rd, Godfrey DJ. Digital x-ray tomosynthesis: current state of the art and clinical potential. Phys Med Biol. 2003; 48(19):R65-R106. [PubMed: 14579853]

2. Poplack SP, et al. Digital Breast Tomosynthesis: Initial Experience in 98 Women with Abnormal Digital Screening Mammography. Am. J. Roentgenol. 2007; 189(3):616-623. [PubMed: 17715109]

3. Ren B, et al. Design and performance of the prototype full field breast tomosynthesis system with selenium based flat panel detector. Proc. SPIE. 2005; 5745(1):550-561.

4. Yang G, et al. Stationary digital breast tomosynthesis system with a multi-beam field emission x-ray source array. Proc. SPIE. 2008; 6913(1):69131A.

5. Zhou, O., et al. Stationary x-ray digital breast tomosynthesis systems and related methods. U.S. Patent. 7,751,528. 2010.

6. Liu ZJ, et al. Carbon nanotube based microfocus field emission x-ray source for microcomputed tomography. Applied Physics Letters. 2006; 89(10) 
7. Modinos, A. Field, thermionic, and secondary electron emission spectroscopy. New York: Plenum Press; 1984.

8. Zhang J, et al. Stationary scanning x-ray source based on carbon nanotube field emitters. Applied Physics Letters. 2005; 86(18)

9. Qian X, et al. Design and characterization of a spatially distributed multibeam field emission X-ray source for stationary digital breast tomosynthesis. Med Phys. 2009; 36(10):4389-4399. [PubMed: 19928069]

10. Zhou W, et al. Multi-beam x-ray source breast tomosynthesis reconstruction with different algorithms. Proc. SPIE. 2010; 7622(1):76220H.

11. U.S. Food and Drug Administration. Mammography Facility Surveys, Mammography Equipment Evaluations, and Medical Physicist Qualification Requirements under MQSA.

12. Teertstra HJ, et al. Breast tomosynthesis in clinical practice: initial results. Eur Radiol. 2009; 20(1): 16-24. [PubMed: 19657655]

13. Zhang $\mathrm{J}$, et al. Multiplexing radiography using a carbon nanotube based X-ray source. Applied Physics Letters. 2006; 89(6):064106. 


\section{X-ray source array}

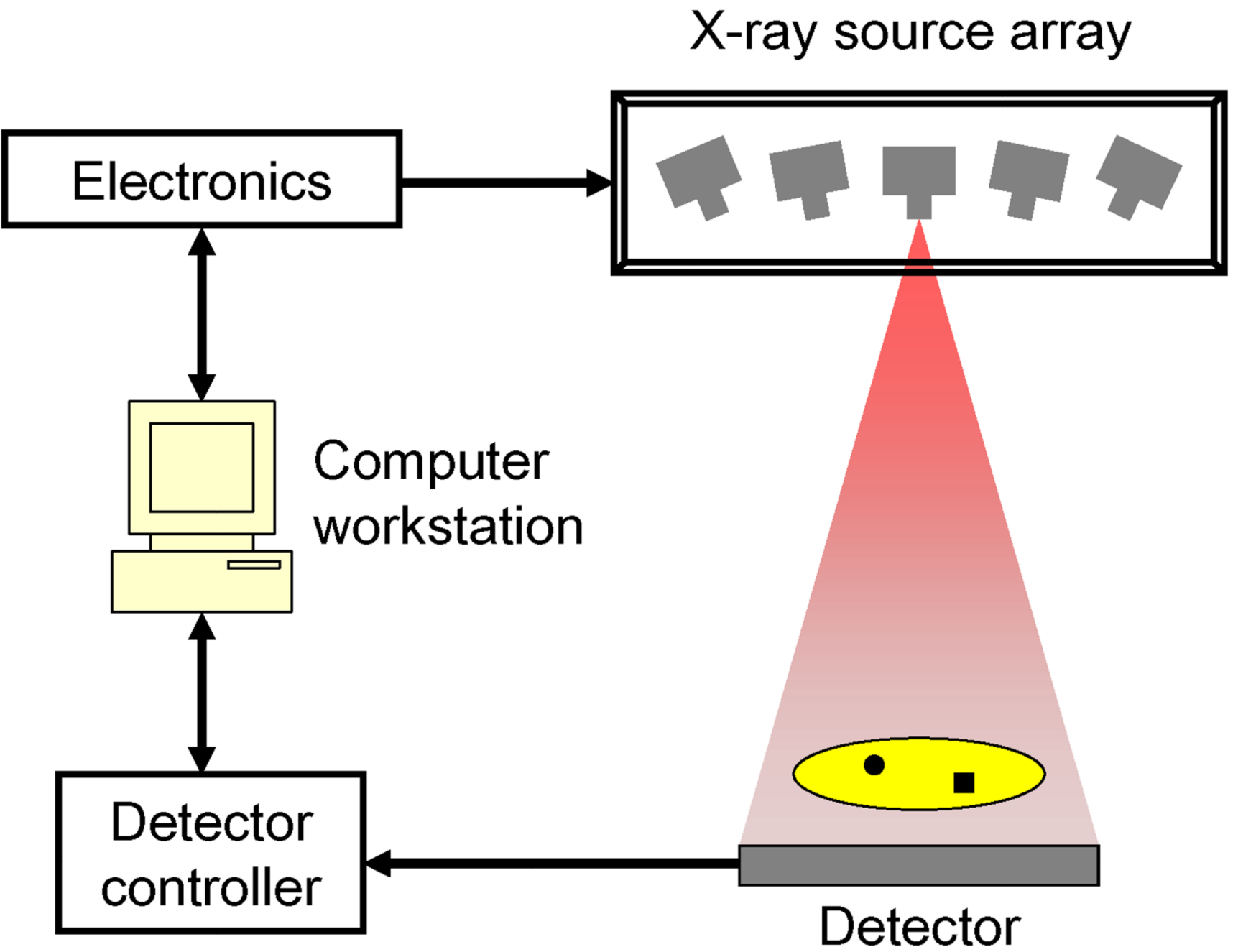

Fig. 1.

Schematic of s-DBT system. 


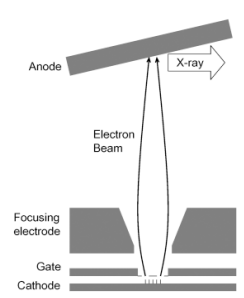

Fig. 2.

Schematic of field emission $\mathrm{x}$-ray source. 


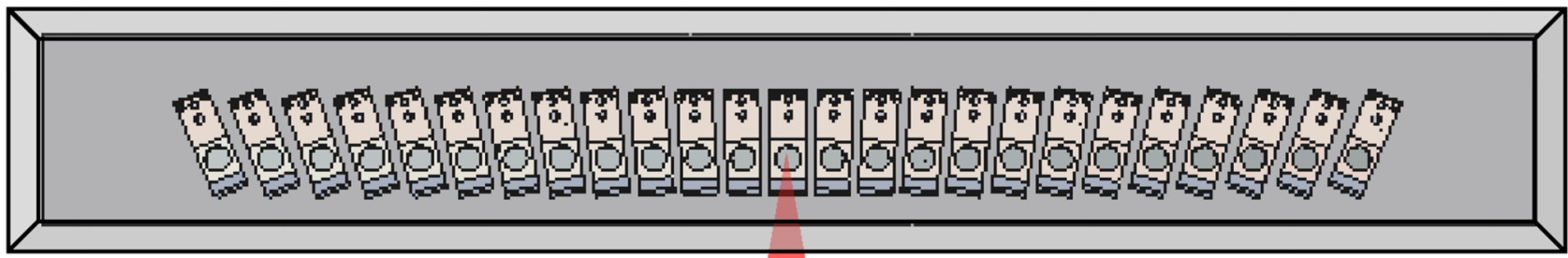

\section{X-ray source array}

\section{Detector}

\section{Breast} phantom

Fig. 3.

Schematic of the bench-top s-DBT system and x-ray source array CAD drawing. 


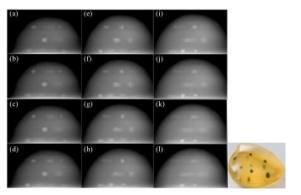

Fig. 4.

Reconstructed slice images of a breast phantom. Slices are $3 \mathrm{~mm}$ apart. The picture of the phantom shows the masses embedded inside. 


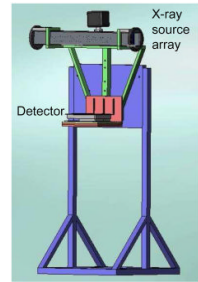

Fig. 5.

CAD drawing of the s-DBT scanner for clinical application. 


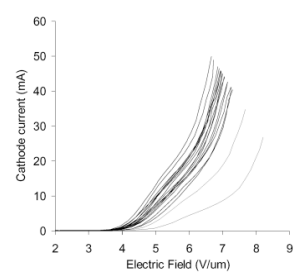

Fig. 6.

Field emission measurement of 19 cathodes with $2 \mathrm{~mm} \times 8 \mathrm{~mm}$ emission area. 